\title{
Training to fit intrauterine devices/intrauterine systems for general practitioners: is there an alternative method of service delivery?
}

\author{
Deborah J Lee
}

\begin{abstract}
Background and methodology This paper questions the traditional method of obtaining intrauterine device (IUD) and intrauterine system (IUS) training, by highlighting the pitfalls of this training, and introduces community IUD/IUS training, a new model offering significant advantages.
\end{abstract}

Discussion and conclusions Traditional IUD/IUS training is not optimal for a variety of reasons including scarcity of designated IUD/IUS clinics, long distances for travel to be trained, wasted clinic appointments, a tendency towards difficult IUD/IUS fitting in these specialist clinics, and a lack of suitable doctors as IUD/IUS trainers. Community IUD/IUS training enables the trainee to be involved in patient selection, setting up an IUD/IUS clinic (probably for their own future use) and following up their own patients. Community IUD/IUS fitting has definite advantages and much to commend it.

Keywords general practitioner, intrauterine device, intrauterine system, long-acting reversible contraception, training

J Fam Plann Reprod Health Care 2007; 33(3): 205-207

(Accepted 23 August 2006)

\section{Introduction}

Specialist methods of contraception such as the intrauterine device (IUD), the Mirena ${ }^{\circledR}$ intrauterine system (IUS) and the subdermal implant, Implanon ${ }^{\circledR}$, are highly effective, long-acting, reversible methods of contraception (LARC). Experience with these methods is highly favourable, and they have the lowest failure rates of all methods of contraception (apart from vasectomy). The 2005 National Institute for Health and Clinical Excellence (NICE) LARC guideline ${ }^{1}$ endorsed the use of these methods in the UK, concluding that these methods are cost effective for the nation, even at 1 year of use.

With a continuing increase in the rate of legal abortions in England and Wales (approximately 5\% in the past 5 years), these excellent concordance-free methods may be at least part of the key to the reversal of these statistics. Yet although patients frequently visit their general practitioners (GPs) about family planning issues, the IUD/IUS are contraceptive methods for which GPs are finding it difficult to obtain training. A study of GP views on IUDs achieved only a $35 \%$ response rate, but of the respondents $34 \%$ did not fit IUDs and only $10 \%$ fitted more than 30 devices per year. ${ }^{2}$ Moreover, the emergency IUD may be difficult to obtain in many GP practices and has been labelled "an endangered species". 3

This paper explores current methods of obtaining IUD/IUS training and describes an alternative model that represents an attractive option to others involved in providing family planning training. A MEDLINE search conducted by the author for the period 1966 to May 2006, which combined searches for intrauterine device(s), IUD with education, medical, (post)graduate, teaching or training, reveals virtually no relevant publications.

\section{Southampton Contraception \& Sexual Health Service,} The Quay to Health, Southampton UK

Deborah J Lee, MFFP, MRCGP, Associate Specialist in Reproductive Health, Lead Doctor for Medical Education and Training

Correspondence to: Dr Deborah Lee, Southampton Contraception \& Sexual Health Service, The Quay to Health, 27 Harbour Parade, Southampton, Hampshire SO15 6BA, UK E-mail debbie.lee@scpct.nhs.uk

\section{Key message points}

- Many difficulties exist for general practitioners (GPs) trying to obtain intrauterine device (IUD) training in the traditional way.

- An alternative community IUD training mechanism has evolved in Wessex, which has numerous advantages.

- This model of training may provide a realistic and costeffective alternative for GPs wishing to undergo IUD training.

\section{How are GPs currently being trained to use IUDs/IUS?}

GPs wishing to fit IUDs/IUS are recommended to follow the training programme laid down by the Faculty of Family Planning and Reproductive Health Care (FFPRHC) ${ }^{4}$ and obtain the Letter of Competence in Intrauterine Techniques (LoC IUT). The present paper focuses on IUD/IUS training.

The National Strategy for Sexual Health and HIV 5 lists provision of IUDs, the IUS and implants as Level 2 services. The commissioning toolkit that supports implementation of the National Strategy has a 'service policy' section. Providers of contraception are clearly informed that "all clinicians offering IUD and implant services should be trained to the competencies expected by the Faculty of Family Planning Letters of Competence, or their equivalent, laid down by an educational body, and show evidence of keeping up to date". 5

The FFPRHC trains its own Instructing Doctors; they possess what is currently known as the Letter of Competence of Medical Education (LoC MEd). These Instructing Doctors will be IUD/IUS trainers, normally within the setting of a community family planning clinic (FPC).

The Faculty LoC IUT programme states that doctors wishing to have IUD/IUS/implant training must already possess an in-date Diploma of the Faculty of Family Planning (DFFP) and must undergo re-accreditation every 5 years. 4

To be IUD/IUS trained, traditionally the training GP attends a number of FPCs (preferably designated IUD/IUS/implant clinics) in order to acquire the practical skills necessary for safe independent practice. The 
IUD/IUS training programme is competency based, with a minimum of seven IUD insertions needed in order to obtain the certificate. Training includes knowledge of all currently available IUDs, and competence in fitting at least two different devices, of which one must be a copper IUD (i.e. training cannot solely comprise fitting the Mirena IUS).

\section{What difficulties are occurring with traditional IUD/IUS training?}

A number of difficulties associated with traditional IUD/IUS training have been identified as follows:

- The national propensity for teenage FPCs - teenagers being a group that would not usually use IUDs means that there are fewer clinics to act as GP training venues.

- Many small family planning services do not have a designated IUD/IUS/implant clinic.

- GPs wishing to learn to fit IUDs may face long waiting lists, sometimes of up to 2 years. DFFP candidates may take priority for training.

- Large cities have larger, more comprehensive family planning services and GPs can access clinics with relative ease. Rural areas, however, present a more difficult problem for patients and doctors alike.

- To obtain training GPs have to leave their surgery, travel long distances (occasionally up to 100 miles) and perhaps pay a locum. At the IUD/IUS clinic there may, for example, be unsuitable patients and/or those who fail to attend their appointment, and the GP concerned may end up doing no IUD fittings. This is dispiriting and consequently many doctors give up trying.

- IUD/IUS clinics are difficult to run. With a 30minute appointment required for an IUD/IUS fitting, if patients choose not to attend there can be much time wasted. Typically, patients sometimes arrive at the clinic (despite being advised otherwise) at unsuitable times in their cycles for IUD/IUS fitting. After counselling, some may change their minds.

- Patients may be referred to FPCs following previous failed attempts at fitting, meaning that these can be difficult IUDs to fit and not necessarily ideal ones for training purposes.

- The training GP is not involved in patient selection or follow-up.

- Training takes place in the FPC, where there are the correct instruments, good lighting and appropriate tools for microbiological testing. This may not be the case when the doctor returns to fit IUDs in their own practice.

- The training GP may not see the same Instructing Doctor. This may be deleterious since the new Instructing Doctors may feel the trainee should watch their own personal technique, resulting in missed opportunities for fittings.

\section{Community IUD/IUS training}

Because of these problems, a new system of training for IUD/IUS began in Wessex in 2000. Set-up involved negotiation with the local family planning service, the GPs themselves, the local Primary Care Trust (PCT) and the pharmaceutical company, Schering Health Care. Although the community IUD/IUS training mechanism described below has been recognised as an option in the past on an $a d$ hoc basis, the author is not aware of the existence of any similar community IUD/IUS/implant training service in operation.
How does community IUD/IUS training work?

The training GP organises an IUD/IUS clinic within their own surgery. The FFPRHC Instructing Doctor attends the clinic at the GP practice and supervises the training (i.e. the training is conducted within the GP's own surgery using the GP's own patients).

\section{How is the community IUD/IUS training service organised?}

Clinics are set up within the GP surgery, with 30-minute appointments, and between four and six appointments are booked per session (maximum of six).

Patients have been identified, counselled and given a Family Planning Association (FPA) leaflet about the IUD and the IUS before they attend the in-house IUD/IUS clinic. They also receive written instructions, including the fact they must use effective contraception until the day of the IUD/IUS fitting. Reception staff are also appropriately trained.

A nurse's presence ensures patient comfort and advocacy, provides assistance with setting up trolleys and sterilising equipment, and also facilitates nurse training. The patient may well re-attend or phone the practice nurse after their IUD/IUS fitting.

Each training doctor is given an IUD training folder containing information about IUD/IUS, setting up clinics, clinical governance stickers/proformas, FPA leaflets and FFPRHC training forms. Basic equipment like Instillaquills ${ }^{\circledR}$ and plastic sounds are also provided.

There is an initial 30-minute pre-clinic assessment including demonstration of the IUD/IUS fitting procedure on a plastic model. The first patient is always seen by the Instructing Doctor for counselling and fitting, and is observed by the trainee. The trainee will practise inserting a number of different IUDs using a plastic pelvis. Ideally at least one example of each device is fitted during training, usually T-Safe $\mathrm{Cu} 380 \mathrm{~A}^{\circledR}$, Nova T380 ${ }^{\circledR}$ and the Mirena IUS. Trainees are made aware of all currently available devices. All patients are given a letter containing postfitting instructions and are asked to return for a 6-week check.

FFPRHC training forms are completed during training, in addition to post-training feedback and evaluation.

\section{Why is community IUD/IUS/implant training advantageous?}

Community IUD/IUS/implant training is advantageous for a number of reasons as follows:

- The system works best if the whole practice is involved in recruiting patients for the IUD/IUS clinic. A Contraceptive Update presentation preceding the onset of training raises awareness of contraception for the primary care team.

- Patients and doctors do not have to travel long distances to attend their FPC.

- IUD/IUS insertions tend to be the easier ones from a technical point of view.

- Equipment availability is checked against the FFPRHC checklist. This may involve ordering some new instruments, which are therefore available within the practice for future use.

- Clinical governance stickers/proformas are used with each procedure. Templates may be produced from these to record IUD/IUS fitting data on the practice computer.

- Practice nurses also benefit from this family planning in-house activity.

- The GP is supported practically and financially with the clinic information pack, textbook and instruments. 
- The GP does not have to leave the practice, pay a locum or travel.

- Patients know and trust their own GP and seem less likely to fail to attend.

- Family planning issues within the practice may come to light, leading to further improvements in patient care.

- Training costs are partially reimbursed by the PCT, and also by a pharmaceutical company, making training financially viable for GPs. Funding from a number of postgraduate educational bodies is currently available from which GPs may claim some reimbursement.

- Doctors motivated to participate in this type of training are those who go on to use it. Within a year of completing community IUD/IUS training, one doctor has fitted more than 60 devices and another approximately 40 .

- Other practice contraceptive training issues can be addressed in house (e.g. subdermal implant training).

- The same Instructing Doctor tends to conduct the training, thus providing continuity, which trainees have reported as beneficial.

- Training is frequently completed in three sessions, whereas it may take longer if the training doctor has to attend an FPC (particularly if there is no designated IUD/IUS clinic and the training doctor is having to attend the general family planning sessions.)

\section{What are the disadvantages of community IUD/IUS training?}

Disadvantages of community IUD/IUS training include:

- The training GP has to make a considerable effort to recruit patients. Empty clinics waste time (this is a problem that also occurs in FPCs). Usually, however, once the practice team understand how the clinics operate then recruitment is not a problem, rather there are not enough clinic slots available to satisfy demand.

- In family planning IUD/IUS clinics, patients may be referred with problems (e.g. lost threads, Actinomycosis) and these learning opportunities simply

Box 1: Faculty of Family Planning and Reproductive Health Care (FFPRHC) changes to fitting the intrauterine device/intrauterine system

Since this article was written, the FFPRHC has made a number of changes that facilitate obtaining a Letter of Competence in Intrauterine Techniques (LOC IUT) as detailed below.

- The training must be supervised overall, and signed off by a primary trainer, who must be a Faculty Instructing Doctor, and have an in-date LoC IUT themselves. The primary trainer can then delegate training to an appropriately experienced doctor to be the secondary trainer. The secondary trainer must have an in-date LoC IUT, but does not need to possess LoC MEd (e.g. this could be another GP in the practice). This means that the primary trainer may only need to do an initial assessment and a final assessment, but the bulk of training can be supervised more easily.

- An amnesty has been put in place whereby any doctor with lapsed DFFP membership payments can get back on board without paying the whole backlog of unpaid subscriptions. This means that so long as a doctor meets the recertification criteria in terms of hours of continuing medical education (CME), they can rejoin the Faculty with ease, pay the current year's subscription only, and move forward swiftly to obtaining an LoC IUT, without necessarily having to do the full DFFP all over again.

- 'Experienced' doctors are permitted to self-certify up to five IUD/IUS fittings for their LoC IUT. This means that as the minimum number of IUD/IUS now required for the certificate is seven, the Instructing Doctor may only need to observe a minimum of two fittings (e.g. one copper IUD and one Mirena IUS), depending on the level of competency. may not present in general practice. A multiple-choice questionnaire has been devised to ensure that these key issues have been discussed. As a result, the checklist on the FFPRHC IUD training form for these topics can thus be completed.

- The Instructing Doctor has to travel to and from the clinic.

\section{Conclusions}

Addressing the family planning training needs of GPs within the primary care setting in which they work, rather than in the community FPC, has thus been shown to have benefits. The advantages clearly outweigh the disadvantages. There are numerous ways in which this training set-up has resulted in rapid, convenient training sessions taking place, ensuring patients are able to obtain their contraceptive method of choice, as well as benefiting their GP in acquiring new skills.

The new technique of bringing the family planning trainer to the practice can be seen as a positive step, and one that erodes the barriers that still exist in some areas between GPs and family planning services. It has also demonstrated how the pharmaceutical industry can work to support training and directly benefit patient care. (NB. Training involves all types of LARC and is not restricted in any way to any one individual sponsor's products.)

IUD/IUS fitting is a much-needed skill for primary care; indeed there is a deficit of IUD fitters and moreover only a small number of doctors qualify as IUD trainers. Recognising the skills of experienced family planning doctors is important. This is one way in which these Instructing Doctors are able to meet training needs outside the realm of any (few) IUD/IUS training clinics.

GPs who have been though this training have spoken highly of it (the results of a qualitative community IUD/IUS training survey will be published separately). To date, more than 35 doctors have benefited from both community IUD/IUS and implant training.

\section{Author's note}

Journal readers should note that the FFPRHC advice on fitting the IUD/IUS has changed since this article was written in 2006. Box 1 contains further information on the relevant changes.

\section{Acknowledgement}

The author wishes to acknowledge the assistance of Jane Chrystal of Schering Health Care who was integral in developing this training programme and without whom none of this would have been possible.

\section{Statements on funding and competing interests}

Funding None identified.

Competing interests The author has worked for several drug companies including Schering and Organon in a training and educational capacity.

\section{References}

1 Faculty of Family Planning and Reproductive Health Care website. http://www.ffprhc.org.uk [Accessed 3 September 2006].

2 Department of Health. National Strategy for Sexual Health and HIV. 2001. http://www.dh.gov.uk [Accessed 3 September 2006].

3 National Institute for Health and Clinical Excellence (NICE). Long-acting Reversible Contraception: The Effective and Appropriate Use of Long-acting Reversible Contraception (NICE Clinical Guidance CG030). 2005. http://www.nice. org.uk/CG030 [Accessed 3 September 2006].

4 Gupta S, Miller JE. A survey of GP views in intra-uterine contraception. Br J Fam Plann 2000; 26: 81-84.

5 Reuter S. The emergency intrauterine device: an endangered species. J Fam Plann Reprod Health Care 2003; 29(2): 5.

6 Lee DJ. Training to insert an IUD/IUS. Trends in Urology, Gynaecology \& Sexual Health November/December 2005; 10: 32-34. 